\title{
What do we do and how do we do it? Assessing genetic counselling in the modern era
}

\author{
Laura Yeates ${ }^{1,2,3} \cdot$ Alison McEwen ${ }^{4} \cdot$ Jodie Ingles $\mathbb{D}^{1,2,3}$
}

Received: 25 March 2020 / Accepted: 9 April 2020 / Published online: 27 April 2020

(c) European Society of Human Genetics 2020

Genetic counselling is a process that aims to support and educate an individual about the medical, psychological and familial aspects of heritable disease. Genetic counselling encompasses a wide range of tasks, from documenting a family history and assessing risk, providing general education about a condition and inheritance patterns, discussing genetic testing options, and providing psychological support to promote adaptation to the diagnosis or carrier status [1]. As we integrate genomics into mainstream healthcare, growing numbers of healthcare professionals and individuals will be impacted. With increasing demand, evaluation of the outcomes of genetic counselling are needed to build a robust evidence base on which to further develop our field. Determining what those outcomes are, and patient-reported outcome measures (PROMs) to effectively assess these then becomes increasingly necessary.

In this issue, Voorwinden et al. report outcomes of a large population of patients attending a genetic counselling service [2]. Using the Dutch versions of three well validated PROMs: Genetic Counselling Outcomes Scale (GCOS), the perceived personal control (PPC) questionnaire and the short form of the State-Trait Anxiety inventory (STAI); they report outcomes of patients attending two services for genetic counselling. Overall, all three outcomes; empowerment, PPC and anxiety, improved after genetic counselling. Demographic and clinical variables were assessed at group and individual levels for

Jodie Ingles

j.ingles@ centenary.org.au

1 Cardio Genomics Program at Centenary Institute, The University of Sydney, Sydney, NSW, Australia

2 Faculty of Medicine and Health, The University of Sydney, Sydney, NSW, Australia

3 Department of Cardiology, Royal Prince Alfred Hospital, Sydney, NSW, Australia

4 Graduate School of Health, University of Technology Sydney, Sydney, NSW, Australia association with the genetic counselling outcomes. At an individual level, a significant proportion of respondents remained stable (42\% on empowerment, $66 \%$ on perceived control, $76 \%$ on anxiety) or indeed worsened on all outcomes after genetic counselling (10\% on empowerment, $13 \%$ perceived personal control, $7 \%$ on anxiety). In understanding why some do worse, the authors show that the type of genetic result was associated with greater levels of anxiety, however, no other risk factors were identified.

An interesting question is therefore raised. How could genetic counselling have been more effective in these families who show worse outcomes? Whether these individuals require more time to adapt and would subsequently improve over time is not known. We cannot disregard the fact that for some participants, even with excellent genetic counselling, adaptation to their diagnosis may not result in better outcomes. For some the future will hold continuing uncertainty about medical outcomes and reconsideration of their imagined future. Importantly though, robust and validated PROMs could flag those patients who require additional support and where genetic counselling may need to be further tailored.

\section{Goals of genetic counselling}

To truly understand whether the goals of genetic counselling are being met, we need to consider the breadth of genetic counselling competencies that may be covered in a session. Practice models including the reciprocal engagement model have been developed, which encompasses a "mutual process in which the genetic counsellor and patient participate in an education exchange of genetic and biomedical information shaped by their unique psychosocial identities" [3]. A wide range of competencies may be covered in a genetic counselling session, from education, to risk assessment and psychological response. The required genetic counselling skills will differ from counselee to counselee, even amongst those referred for the same reason. Therefore, the broad scope of practice in genetic 
counselling requires equally broad assessment tools that can adequately evaluate such variable needs.

There is significant international variation in both service delivery and the training of the health professionals who provide genetic counselling. As we consider the use of PROMs in evaluating genetic counselling practice, it is important to consider how models of genetic counselling and indeed the training of health professionals vary internationally. Genetic counselling is often provided by genetic counsellors, nurses, clinical geneticists and medical social workers. Indeed, as reported by Voorwinden and colleagues, genetic counselling was provided by both genetic counsellors and clinical geneticists. As a process, genetic counselling provides both education and psychological support [1]. While many healthcare professionals are well placed and trained to provide one or the other, fewer are trained to effectively provide both. Health professionals capable of providing effective genetic counselling are likely to be in short supply, and measures to bolster the workforce with additional people, but also to better use technology to support their practice, will be increasingly necessary. In this respect, valid measures of genetic counselling effectiveness will indeed be important in determining the incremental value of such approaches.

\section{Tools to support effective genetic counselling}

Developing clinically usable tools to assist with identifying patients and relatives who are more likely to experience adverse outcomes from genetic counselling may serve to ensure that all are offered appropriate, individualized follow-up. Others have demonstrated the use of PROMs to inform clinical practice. Ison et al. invited patients to complete the GCOS and adapted their genetic counselling session based on the results [4]. Costal Tirado et al. used PROMs to assess the quality improvements in a clinical genetic setting, highlighting health professionals considered PROMs a helpful tool in assessing their service [5]. Taken together, these studies highlight the value of PROMs in assessing the impact of genetic counselling. The additional value of incorporating PROMs into clinical practice as an

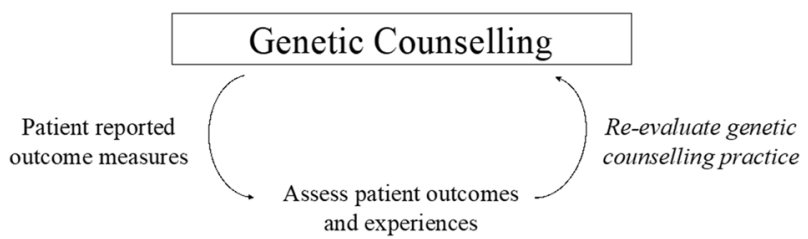

Fig. 1 Patient-reported outcome measures in genetic counselling practice: Evaluating patient reported outcomes from genetic counselling is helpful in assessing impact, but also allowing us to revise our practice to improve outcomes. evaluation tool to assess genetic counselling outcomes is becoming clear (Fig. 1), providing another opportunity to assess genetic counselling outcomes and ensure the core service of a "client-centred approach" is upheld.

\section{Summary}

With the integration of genomics in to healthcare, it is timely to evaluate genetic counselling practice. The need for genetic counselling across many fields of medicine has seen expansion into different practice settings and using a variety of practice models, evaluating what we do and how we do it has never been more important to ensure the core values of genetic counselling are always upheld and patients receive the highest standard of care. PROMs provide a means to evaluate our practice across different healthcare settings, allowing providers to assess gaps in current services or identify patients that require additional support. As an international community, we must continue to develop evidence and advocate for the need for genetic counselling across different service delivery models.

Acknowledgements LY is a recipient of a co-funded National Heart Foundation of Australia/National Health and Medical Research Council (NHMRC) PhD scholarship (\#102568 and \#191351). JI is the recipient of an NHMRC Career Development Fellowship (\#1162929).

\section{Compliance with ethical standards}

Conflict of interest JI receives research grant support from Myokardia, Inc. The other authors declare that they have no conflict of interest.

Publisher's note Springer Nature remains neutral with regard to jurisdictional claims in published maps and institutional affiliations.

\section{References}

1. National Society of Genetic Counselors' Definition Task F, Resta R, Biesecker BB, Bennett RL, Blum S, Hahn SE, et al. A new definition of genetic counseling: National Society of Genetic Counselors' Task Force report. J Genet Couns. 2006;15: $77-83$.

2. Voorwinden JS, Plantinga M, Ausems M, Knoers N, Velthuizen M, Birnie E, et al. Cognitive and effective outcomes of genetic counselling in the Netherlands at group and individual level: a personalized approach seems necessary. Eur J Hum Genet. 2020.

3. Veach PM, Bartels DM, Leroy BS. Coming full circle: a reciprocalengagement model of genetic counseling practice. J Genet Couns. 2007; 16:713-28.

4. Ison HE, Ware SM, Schwantes-An TH, Freeze S, Elmore L, Spoonamore KG. The impact of cardiovascular genetic counseling on patient empowerment. J Genet Couns. 2019;28:570-7.

5. Costal Tirado A, McDermott AM, Thomas C, Ferrick D, Harris J, Edwards A, et al. Using patient-reported outcome measures for quality improvement in clinical genetics: an exploratory study. J Genet Couns. 2017;26:1017-28. 